\title{
EL PAPEL DE LA TRADUCCIÓN EN EL AULA DE ELE DESDE LA PERSPECTIVA DEL ESTUDIANTE UNIVERSITARIO ALEMÁN
}

\section{THE ROLE OF TRANSLATION IN THE ELE CLASSROOM FROM THE PERSPECTIVE OF THE GERMAN UNIVERSITY STUDENT}

\author{
Alicia ESCRIBANO SABIO \\ Universidad de Heilbronn (Alemania)
}

\section{Resumen}

El objetivo de este artículo es reflejar la importancia y la necesidad que el estudiante alemán universitario de nivel inicial le otorga al uso de su lengua nativa dentro del aula. Para conseguir nuestro propósito, hemos llevado a cabo unas encuestas realizadas en clase en las que se puede observar la relevancia del papel preponderante de la traducción en el aula de lenguas extranjeras, concretamente en la enseñanza de español como lengua extranjera.

Palabras clave: perspectiva del alumno, traducción en clase, lengua extranjera, didáctica de lenguas.

\section{Abstract}

This article looks at German university students learning a language at beginner level. Its aim is to reflect on how important and necessary it is for these students to use their native language in the classroom. To achieve our purpose, we carried out classroom surveys, which allowed us to observe how significant a role translation plays 


\section{A. Escribano Sabio}

2020. "El papel de la traducción en el aula de ele desde la perspectiva del estudiante universitario alemán" Sabir. International Bulletin of Applied Linguistics. 1,2: 33-45.

in the foreign language classroom, specifically in the teaching of Spanish as a foreign language.

Keywords: student perspective, classroom translation, foreign language, language learning.

\section{Introducción}

El papel de la traducción como herramienta en el aprendizaje de lenguas extranjeras ha sido objeto de discusión durante décadas, pasando de ocupar un papel preponderante en el método tradicional a ser considerada un instrumento perjudicial en la didáctica de lenguas, especialmente con la llegada del método directo. Sin embargo, en la actualidad se aboga por su uso como actividad complementaria a la didáctica comunicativa de lenguas.

En el presente artículo, nuestro objetivo será llevar a cabo un breve estudio sobre las creencias, perspectivas y opiniones de estudiantes universitarios alemanes sobre el uso de su lengua materna en el aula. El perfil de estos alumnos se corresponde con estudiantes de Economía y Turismo de la Universidad de Heilbronn (Alemania) que tienen la asignatura de Español como Lengua Extranjera ${ }^{1}$ integrada en su plan de estudios representando, de esta manera, una materia de suma importancia. Cursan la asignatura en el primer, segundo y tercer semestre, lo cual equivale al primer y al segundo año de carrera, según los planes de estudios españoles. En el nivel A1 se aprende español dos horas lectivas ${ }^{2}$ a la semana y en el nivel A2 cuatro horas semanales.

Hemos elegido un tipo de encuesta de preguntas cerradas mayoritariamente, mediante las cuales el estudiante podía marcar lo que piensa acerca del uso de su lengua materna en el aula de ELE de manera más rápida.

\footnotetext{
${ }^{1}$ A partir de aquí, ELE.

2 En Alemania una hora lectiva se corresponde con 45 minutos de clase. Se suelen impartir dos clases juntas, es decir, en bloques de 90 minutos.
} 
El tipo de traducción al que aludimos es la traducción pedagógica, herramienta didáctica que se aplica como soporte para la Enseñanza de Lenguas Extranjeras en el aula y que aporta ciertas ventajas:

The proper use of pedagogical translation can show how this activity is not uncommunicative and that it does not merely focus on accuracy. Translation exercises can serve a variety of purposes ranging from linguistic problems to more cultural, semantic and pragmatics concerns. Furthermore, translation can help learner enhance their analytical and problem-solving skills which are essential in everyday life as well as in most working fields.

(Leonardi, 2010: 22)

\section{La traducción en la historia de la enseñanza de lenguas}

El uso de la traducción en clase se ha visto envuelto en polémica a lo largo de décadas. Si nos remontamos al método tradicional, se incluye un determinado elenco de características, cuyos rasgos definitorios presentamos a continuación:

- La elaboración del currículo sobre el eje de una descripción gramatical de la lengua.

- El predominio de la gramática normativa en el conjunto de objetivos que deben alcanzarse. (...).

- La memorización de listas de vocabulario.

- La presencia de cada lección de temas de traducción directa e inversa.

(Sánchez Iglesias, 2009: 33). 


\section{A. Escribano Sabio}

2020. "El papel de la traducción en el aula de ele desde la perspectiva del estudiante universitario alemán" Sabir. International Bulletin of Applied Linguistics. 1,2: 33-45.

Como podemos observar, este método abogaba por el uso de la traducción en su gama de actividades, otorgándole a esta estrategia un papel muy importante. Sin embargo, con la llegada del método directo, como hemos mencionado anteriormente, el papel de la traducción en clase se vio totalmente desterrado.

Posteriormente, con la llegada del método audio-oral ocurrirá lo mismo que con el método directo, la traducción estará terminantemente prohibida:

La traducción está desaconsejada o prohibida porque se parte del convencimiento de que el uso de la lengua materna propicia las interferencias y resta eficacia al aprendizaje de una segunda lengua. De ahí que el ideal resida en lograr que los alumnos "se olviden" " de su propia lengua. Solo se permite que el profesor recurra a gestos u otros signos no lingüísticos para lograr que los alumnos entiendan las palabras o estructuras.

(Sánchez, 2009: 75)

En nuestros días nos inclinamos a una metodología que haga primar la comunicación, posición que desemboca en una crítica continua a las actividades de traducción, debido a que son consideradas como actividades "no comunicativas". Sánchez Iglesias opina que "la traducción constituye un medio y no un fin, además de que el enfoque comunicativo no se ha manifestado nunca en contra de esta herramienta". (Sánchez Iglesias. 2009: 36, 37).

Este autor nos resume, brevemente, la adaptación de la traducción a los niveles iniciales e intermedios de Newmark:

\section{Nivel inicial:}

- Cerciorarse de que los alumnos no están asumiendo significados incorrectos de determinados términos en la L2.

\footnotetext{
${ }^{3}$ Comillas de autor.
} 


\section{A. Escribano Sabio}

2020. "El papel de la traducción en el aula de ele desde la perspectiva del estudiante universitario alemán" Sabir. International Bulletin of Applied Linguistics. 1,2: 33-45.

- Consolidación de la parte gramatical y léxica.

- Traducción literal de algunas expresiones de L2 para facilitar su comprensión y memorización.

Nivel intermedio:

- Traducción directa de palabras o frases en el tratamiento de algunos errores, llamando la atención sobre determinadas interferencias lingüísticas.

- Ampliación de vocabulario de la LO, especialmente cuando se trate de aspectos de sinonimia en un determinado caso semántico.

(Newmark. 1992).

Capel Moreno justifica la práctica de la traducción como complemento de otras estrategias unilingües:

- Permite trabajar desde la LM del alumno, lo que supone el desarrollo de la capacidad de expresión en LE y hacia la LM del alumno, lo que requiere la capacidad de comprensión en LE.

- Invita a la discusión y especulación, y por lo tanto se puede considerar una actividad comunicativa que fomenta el desarrollo de estrategias de aprendizaje.

(Capel Moreno. 2005: 402)

\section{La influencia de la lengua materna en el aprendizaje de una lengua extranjera}

A pesar de que muchos métodos han excluido por completo la traducción en el aula de lenguas extranjeras, vista como perjudicial, hay que reconocer que nos guste o no, el estudiante siempre hará uso de su lengua materna, especialmente en los niveles iniciales de la lengua. Pastor Cesteros (2004: 35)la considera una necesidad tan antigua como el nombre, que sin esta no habría sido posible transmitir información 


\section{A. Escribano Sabio}

2020. "El papel de la traducción en el aula de ele desde la perspectiva del estudiante universitario alemán" Sabir. International Bulletin of Applied Linguistics. 1,2: 33-45.

entre las diversas lenguas durante el contacto entre pueblos distintos y la historia de la cultura.

Hurtado Albir nos habla de la traducción interiorizada:

Por traducción interiorizada nos referimos a la estrategia espontánea que utiliza quien aprende una lengua extranjera, de confrontar con su lengua materna lengua y estructuras, para comprender mejor, para consolidar su adquisición, etc; esta estrategia se manifiesta sobre todo al principio del aprendizaje y, a medida que la lengua extranjera va consolidándose, va desapareciendo.

(Hurtado Albir, 1999: 3)

El planteamiento de Hurtado, no va en detrimento de reconocer una casuística muy heterogénea en la realidad del aula de lenguas extranjeras. Unas veces se sigue la dinámica que sigue Hurtado, pero no siempre es así, puesto que hay múltiples tipos de reacciones por parte de los estudiantes (García Marcos, 2018).

\section{Muestras y resultados recopilados.}

Tras nuestro resumen teórico sobre las ventajas y desventajas de la traducción en la enseñanza de una lengua extranjera, pasamos ahora a la siguiente parte de este artículo, constituida por las encuestas tomadas de clase a diferentes grupos con distintos niveles. Las muestras han sido realizadas a 40 estudiantes de cada nivel ${ }^{4}$, lo cual se ve representado en el presente estudio. Son estudiantes de Turismo y de Economía, la edad oscila entre 19 y 23 años. Se realizaron diferentes preguntas acerca del uso de la traducción en clase, abarcando el uso de esta herramienta en actividades y en el desarrollo de la clase. A continuación, presentamos los gráficos con sus resultados y una explicación de estos.

\footnotetext{
${ }^{4}$ Niveles A1, A2, B1, según el Marco Común Europeo de Referencia (MCER).
} 


\section{Gráfico 1}

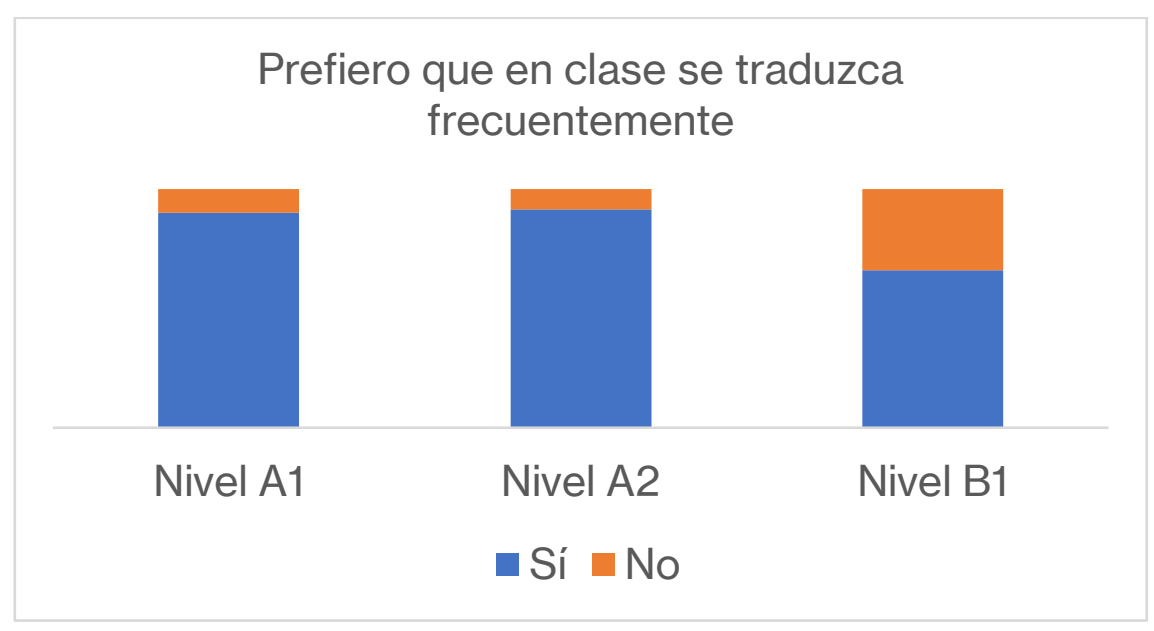

De este gráfico se desprende de manera clara la preferencia de los alumnos a la traducción en clase disminuyendo, como podemos observar, estos resultados cuando se alcanza el nivel B1 de la lengua, lo cual se debe a una mayor seguridad debido a que ya disponen de más herramientas y experiencia con la lengua, imaginándose ya como única lengua usada en el aula la lengua meta.

\section{Gráfico 2}

¿Le parece bien que el libro ofrezca los enunciados solo en la lengua meta?
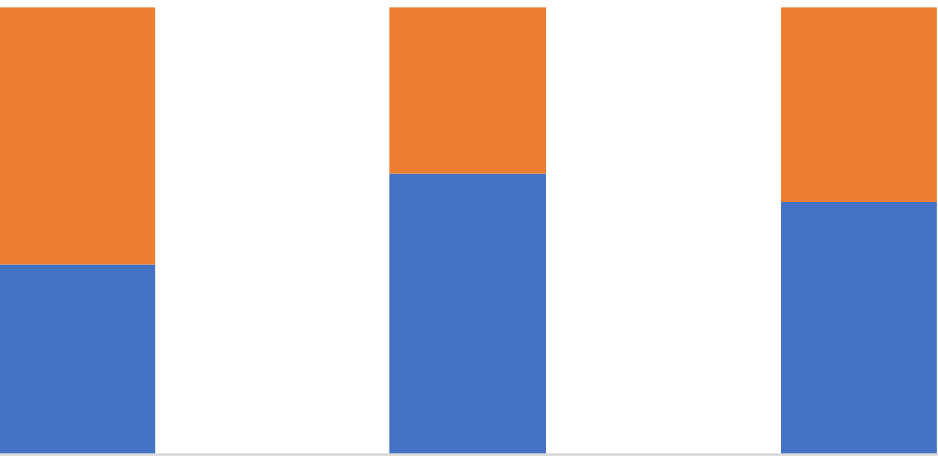

Nivel A1

Nivel A2

Nivel B1

-Sí $\square$ No 
A los alumnos preguntados sobre los enunciados de los ejercicios solo en español, observamos que en los tres niveles en los que se repartió la encuesta no se opone un gran número de ellos, ya que en clase el profesor los traduce, por lo cual no les supone una traba sobre el entendimiento de las instrucciones dadas.

\section{Gráfico 3}

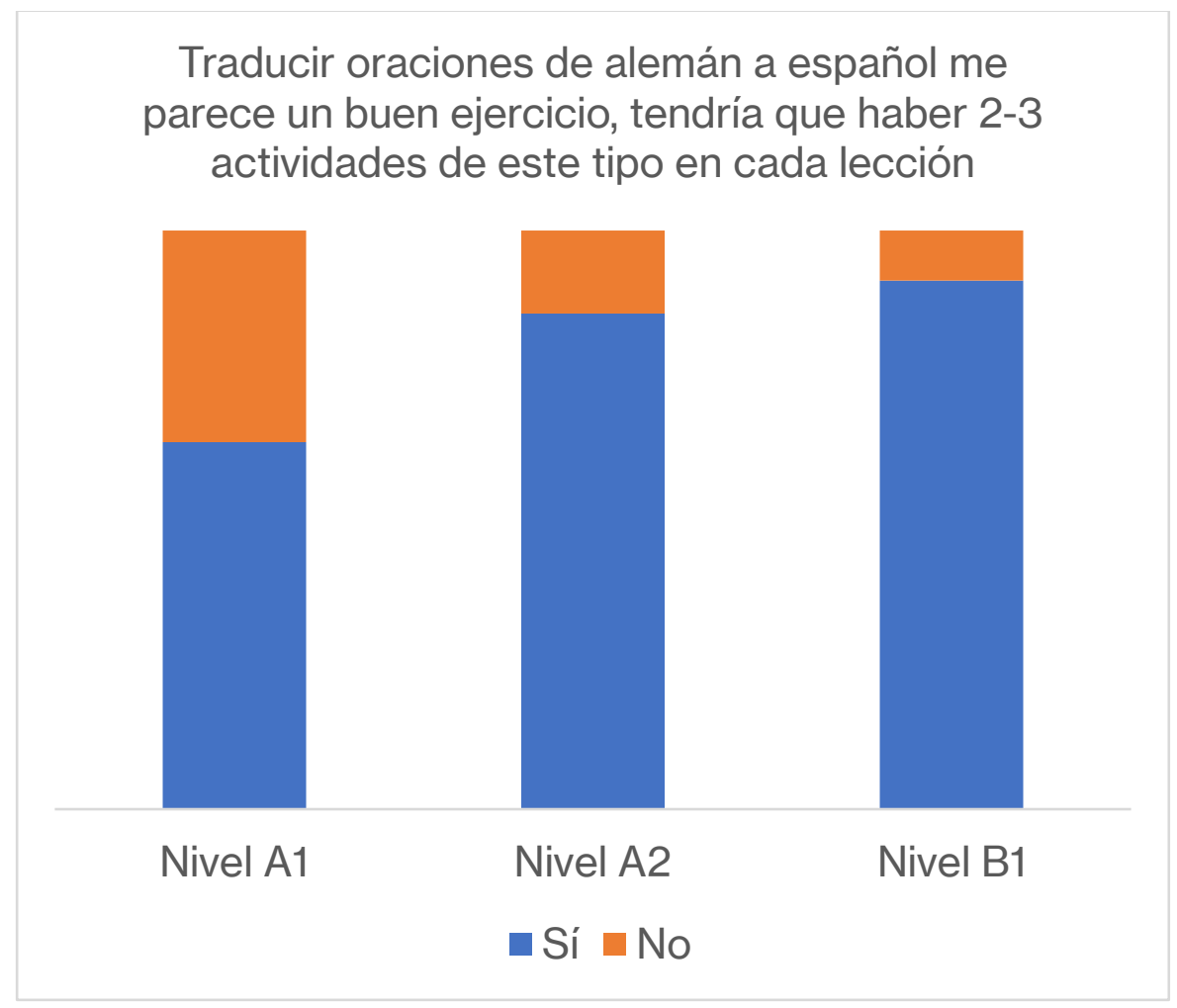

En este gráfico vemos también la aceptación de la traducción en clase, concretamente actividades específicas en las que se traducen oraciones de la lengua nativa a la lengua meta, apoyada esta idea por un gran porcentaje, aumentando esta preferencia en el nivel B1, razón que probablemente se produce por su elevado conocimiento de la lengua en comparación con los niveles anteriores. 


\section{Gráfico 4}

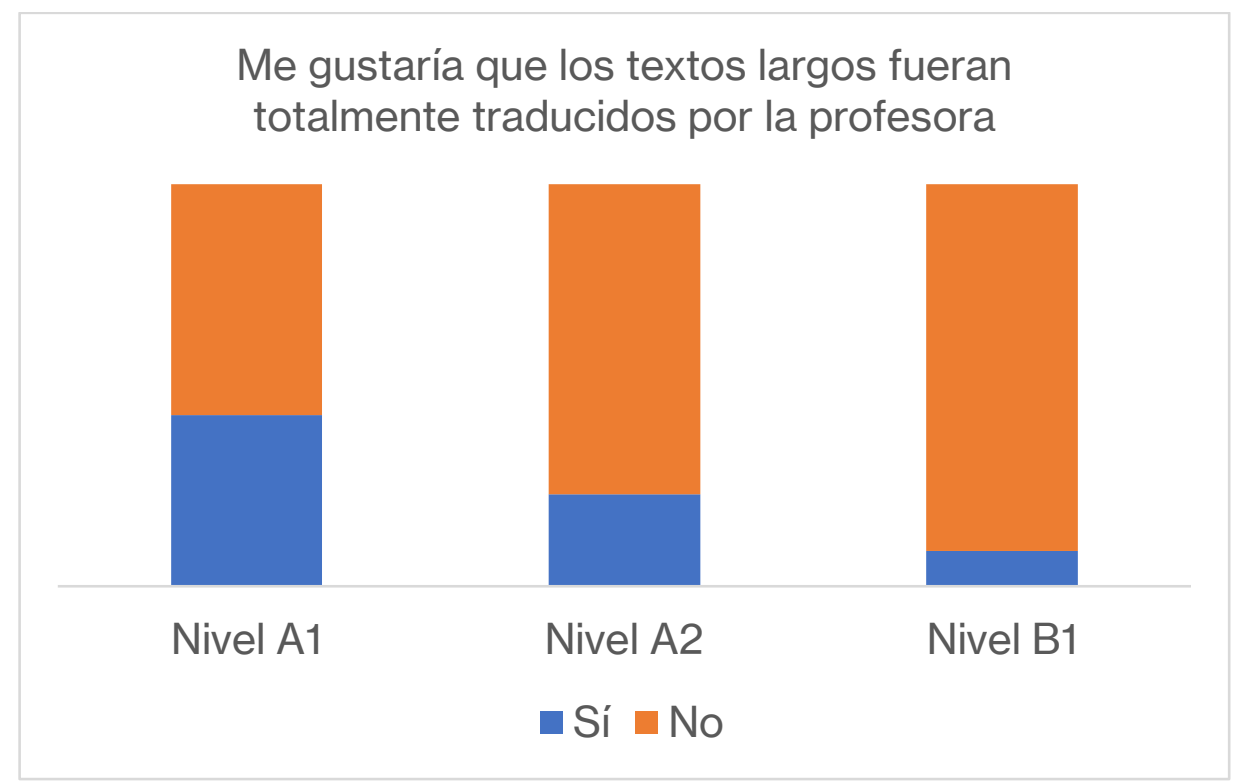

En estos ejemplos se aprecia la preferencia por parte de los discentes del nivel B1 (el más alto de los analizados) por abandonar progresivamente la traducción de textos extensos, sin ser necesaria la traducción del texto por completo, siendo suficiente solo con las palabras desconocidas. Por lo tanto, se ven capaces de poder encontrarle sentido al texto por sí solos y sin basarse en su lengua materna.

\section{Gráfico 5}

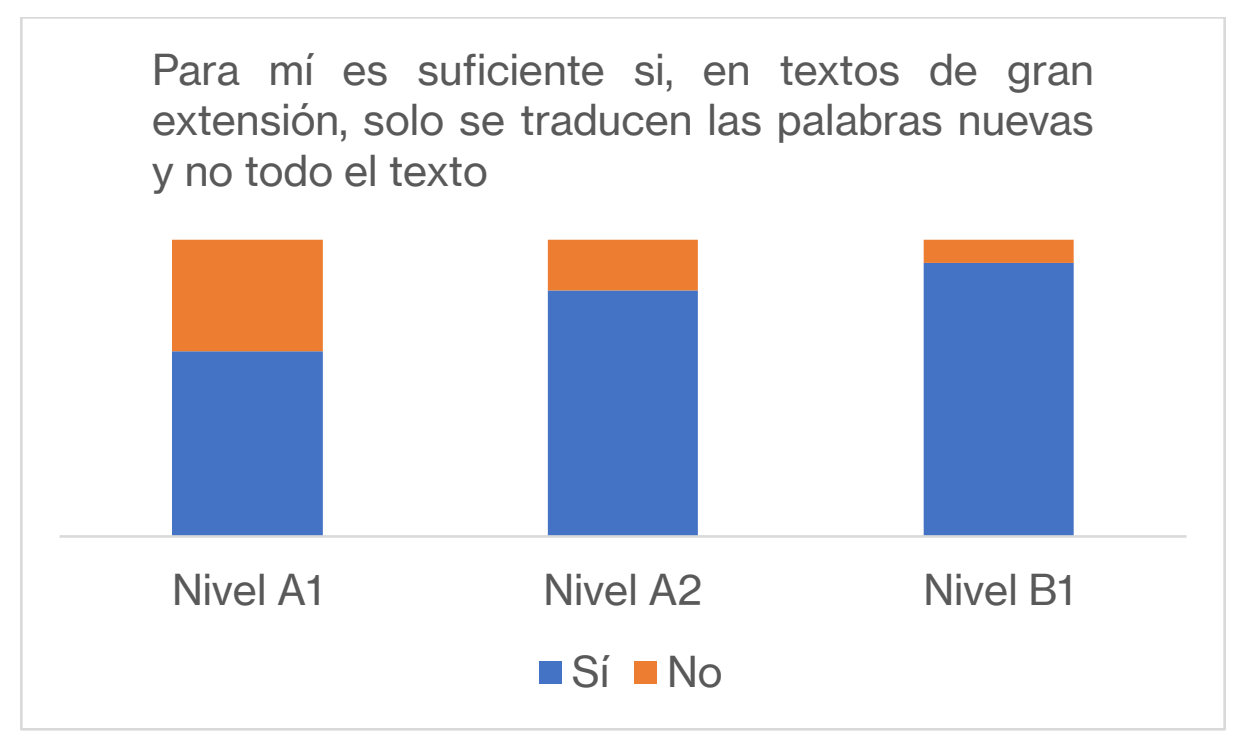

En cuanto a la pregunta que hace referencia a la traducción de textos, se aprecia claramente que no les es suficiente si el docente solo traduce las palabras desconocidas, sin ser necesaria la traducción completa de dichos textos. 


\section{Gráfico 6}

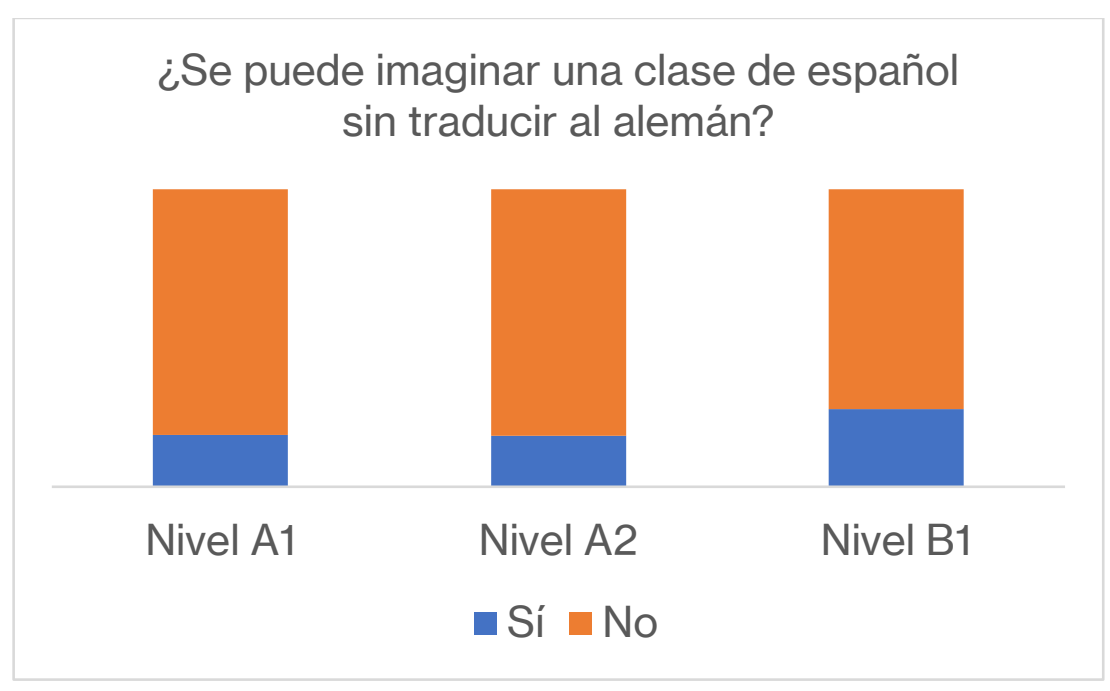

Una clase en la que no se use la traducción en absoluto no parece ser muy aceptada por los estudiantes, mostrándonos los gráficos un claro "no" a la idea de que la clase de ELE tenga lugar solo en la lengua meta, algo que descartan por completo, especialmente en los niveles A1 y A2 dada su poca práctica, experiencia y contacto con la nueva lengua que acaban de empezar a aprender. Ya en el nivel B2 podemos estimar que hay un leve número de más estudiantes que sí se imaginan la clase de ELE solo en la lengua meta, sin apoyo de la lengua materna del estudiante.

\section{Gráfico 7}

¿Podría seguir una clase de español sin que se tradujera nada? Solo mediante imágenes y gestos de la docente.

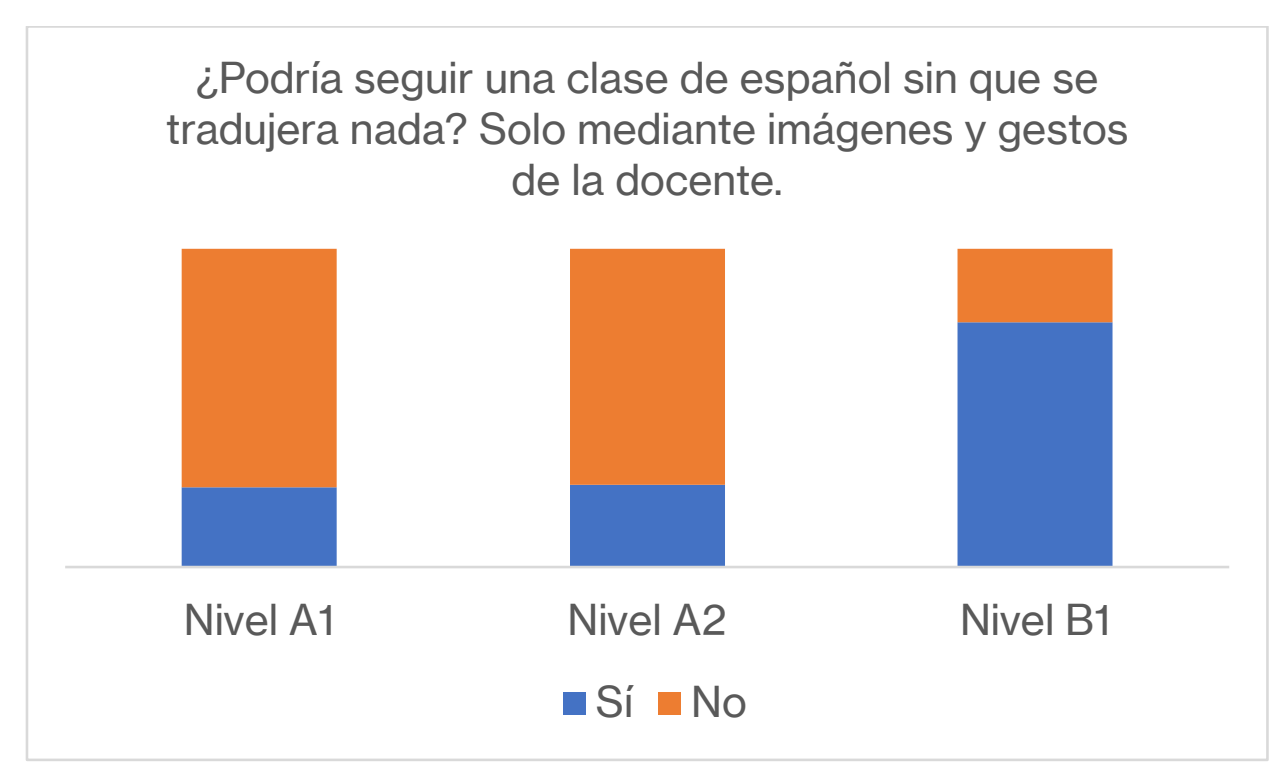


En los dos niveles iniciales los alumnos, en su mayoría, han contestado que serían incapaces de poder continuar una lección en el aula sin la ayuda de su lengua materna, cambiando esta opinión en el nivel B1, en el cual ya se tienen unos conocimientos más sólidos sobre la lengua extranjera que han decidido aprender.

\section{Conclusiones}

Nuestro breve estudio acerca de las perspectivas de los estudiantes en referencia a la traducción pedagógica en clase desprende la importancia de esta en el desarrollo de la clase, siendo muy difícil para ellos si se prescindiera de esta.

No podemos olvidar todas las ventajas que la traducción, a pequeñas dosis, brinda. Mientras se hace uso de esta herramienta cuando la usamos de forma específica en determinadas actividades, se lee y se escribe, lo cual son destrezas lingüísticas apoyadas y defendidas por el MCER.

El uso de la traducción pedagógica en la enseñanza-aprendizaje de una LE puede ser criticado por corresponderse con actividades que no son comunicativas, pero como herramienta de apoyo para aprender mejor las estructuras de la lengua meta y poder comparar en las dos lenguas puede ser de gran utilidad. En los gráficos y en el pequeño estudio realizado a lo largo de este artículo podemos observar que es una actividad y una técnica necesaria para el alumno universitario. Hablamos, en este aspecto, no solo de actividades, sino también del desarrollo de la clase en el día a día, aceptándose y exigiendo, en muchas ocasiones, el uso de su lengua materna, ya que no se puede olvidar que no estamos ante un programa de inmersión, sino que aprenden español en su país.

Aunque mayormente se ha considerado que las actividades de traducción son más idóneas en niveles avanzados, observamos en nuestro artículo que es más aceptado por los estudiantes principiantes que por los de nivel intermedio. Les supone una herramienta de apoyo y de comparación con su lengua nativa y de afianzamiento 


\section{A. Escribano Sabio}

2020. "El papel de la traducción en el aula de ele desde la perspectiva del estudiante universitario alemán" Sabir. International Bulletin of Applied Linguistics. 1,2: 33-45.

en la base gramatical y léxica. Supone también una manera de luchar con las interferencias.

En las explicaciones, por ejemplo, de gramática en clase, el docente tiende a contrastar diferentes puntos gramaticales entre las dos lenguas, perfilando y profundizando las nuevas estructuras que se presentan en el aula.

Como partidaria de la traducción pedagógica en clase, es importante también destacar que es una buena herramienta para contrastar las dos lenguas pero para ello, un punto a destacar es el conocimiento y dominio de las dos lenguas por parte del docente. Podríamos integrarla como una destreza lingüística más.

Este es un campo de investigación en el que aún queda mucho por hacer. Para ello habrá que continuar experimentando en el aula a través de nuestra experiencia y de las perspectivas de los estudiantes en este ámbito para poder, de esta manera, innovar y aprender en referencia a la mejor metodología que podemos usar en el aula.

\section{Bibliografía}

\section{CAPEL MORENO, A, I.}

2008. El diseño de tareas de Traducción en Interpretación: un instrumento en la enseñanza-aprendizaje del inglés como lengua extranjera en bachillerato. Almería: Universidad de Almería. Tesis Doctorales (Edición Electrónica) (Español) CD-ROM - 17 enero 2008.

GARCÍA MARCOS, F.

2018. La trastienda de la enseñanza de lenguas extranjeras. Granada: Comares.

HERNÁNDEZ, M. R.

1996. "La traducción pedagógica en la clase de ELE". Actas del VII Congreso Internacional de ASELE. Consultado el 20-VII-2020, de 
HURTADO ALBIR, A.

1999. Objetivos de aprendizaje y metodología en la formación de los traductores e intérpretes. Madrid: Edelsa.

LEONARDI, $\mathrm{V}$.

2010. The Role of Pedagogical Translation in Second Language Acquisition: From Theory to Practice. Oxford: Peter Lang.

LITTLEWOOD, W.

2012. La enseñanza comunicativa de idiomas. Introducción al Enfoque Comunicativo. Segunda reimpresión, Madrid: Edinumen.

\section{PASTOR CESTEROS, S.}

2004. Aprendizaje de segundas lenguas. Lingüística aplicada a la enseñanza de idiomas. Alicante: Universidad de Alicante.

RICHARDS, J.,C., y RODGERS, T., S.

2009. Enfoques y métodos en la enseñanza de idiomas. Madrid: Edinumen.

SÁNCHEZ IGLESIAS, J.

2009. "La traducción en la enseñanza de lenguas extranjeras: una aproximación polémica”. Biblioteca Virtual RedEle, n 10. Consultado el 18-X-2019, de

http://www.educacionyfp.gob.es/dam/jcr:4efabb0b-47d6-4fae-a9c6a8a13df5fa97/2009-bv-10-22sanchez-iglesias-pdf.pdf

SÁNCHEZ PÉREZ, A.

2009. La enseñanza de idiomas en los últimos cien años. Métodos y enfoques. Madrid: Sgel.

SELINKER, L. y GASS, M., S.

1994. Second language acquisition. An Introductory Course. Segunda edición, New York and London: Taylor \& Francis. 